\title{
Optical Characterization of Gold Nanoparticle Layers Formed on Plastic Microbeads
}

\author{
Hiroshi ShIIGI,*广 Takahiro FuJITA,* Xueling Shan,* Masahiro Terabe,* Atsushi MinashI,* \\ Yojiro Yамамото, ${ }^{* *}$ and Tsutomu NAGAOKA* \\ *Department of Applied Chemistry, Osaka Prefecture University, 1-2 Gakuen, Sakai, Osaka 599-8570, Japan \\ **GreenChem. Inc., 930-1 Fukuda, Sakai, Osaka 599-8241, Japan
}

\begin{abstract}
Generally, the characterization of a metal layer formed on a planar substrate has been achieved using scanning electron microscopy and transmission electron microscopy. These techniques provide details of the surface and/or the crosssection of a planar structure with high resolution. However, the evaluation of sphere-like structures is troublesome owing to the necessity to observe a sample from various angles and/or to calculate the yield from many values obtained for many samples, since the conventional methods can observe a sample only from one direction. We have developed a simple evaluation method for a thin metal layer on plastic microbeads based on its light-scattering properties using dark-field microscopy coupled with a spectrometer. The light-scattering intensity of gold-nanoparticle-coated microbeads depends significantly on the gold coverage. We believe that our study is significant because it describes the development and evaluation of the surface coverage of a thin metal layer on a sphere-like microstructure.
\end{abstract}

Keywords Gold nanoparticle, metal coating, conducting microbead, evaluation of coverage and thickness

(Received October 6, 2015; Accepted January 6, 2016; Published March 10, 2016)

\section{Introduction}

Rapid progress and developments have been made in information technology and its related fields; this has made it necessary to miniaturize compact electronic devices as well as consumer electronic products, such as mobile phones, personal digital assistants (PDAs) and laptops through high-density packaging technology. ${ }^{1,2}$ Consequently, this has led to accelerated development in the metallic thin film technologies over the last few decades. Conducting microbeads have been applied to anisotropic conductive films, which are adhesive insulator films in which conductive particles are dispersed. Conducting microbeads have been obtained through the formation of a thin metal layer on a plastic core microbead using an electroless plating method. The conducting microbeads are also compressed so as to form the connections between the input/output connections and external circuit via a single installation procedure for consumer electronic products through highdensity packaging. Since each micrometer-sized bead enables movement and arrangement at a specific location, microelectronics can be applied to the rapid development of miniaturized compact electronic devices and consumer electronic products, and to electroanalytical uses. ${ }^{3,4}$

Recently, metal nanoparticles (NPs) and their arrays have attracted the attention of researchers as important materials for nano- and micro-electronics owing to their unique optical and chemical properties based on not only the metal species, but also their shapes and sizes. ${ }^{5}$ Moreover, the NPs can be used as

$\dagger$ To whom correspondence should be addressed.

E-mail: shii@chem.osakafu-u.ac.jp building blocks for fabricating micrometer-sized structures, such as dots, wires, and films. ${ }^{6-12}$ Self-assembly technology can be effectively used to assemble well-organized one- to threedimensional microstructures, and inter-particle connections can be controlled at the single-particle level. ${ }^{13}$ Typically, these zeroto two-dimensional microstructures are assessed by using highresolution microscopies, such as scanning probe microscopy (SPM), scanning electron microscopy (SEM), and transmission electron microscopy (TEM). However, it is difficult to evaluate a three-dimensional microstructure appropriately, since it can only be observed from one direction using such a microscope. Here, we propose a new evaluation method for three-dimensional structures using dark-field microscopy (DFM).

We previously reported on gold NP (AuNP) arrays that were prepared via a single-step procedure using self-assembling thiol-terminated molecules to deposit AuNPs on a plastic bead as a micrometer-sized substrate..$^{2-413}$ By applying this method, it is possible to form a thin metal layer consisting of twodimensional films of AuNPs via thiol molecules on the surface of the microbeads. In the present study, we attempted to develop a simple evaluation method to estimate the thickness and surface coverage of a single AuNP layer through thiol binding on a plastic microbead, while focusing on the light-scattering intensity obtained using DFM.

\section{Experimental}

\section{Chemicals}

All of the chemicals used were of reagent grade. Ultrapure water $(>18 \mathrm{M} \Omega \cdot \mathrm{cm})$, after ultraviolet sterilization, was used throughout the experiment. Tetrachloroauric acid $\left(\mathrm{HAuCl}_{4}\right)$ was 
purchased from Tanaka Kikinzoku Kogyo. Sodium citrate, which was used to prepare AuNPs, and thioctic acid, which was used as a binder, were purchased from Wako Pure Chemical Industries. Acrylic resin beads with a mean diameter of $5 \mu \mathrm{m}$ (Hayakawa Rubber Co., Ltd.) were used as the substrates.

\section{Preparation and characterization of AuNPs}

The AuNPs used in this study were prepared using the conventional chemical reduction in aqueous media. AuNPs were characterized using a zeta-potential and particle size analyzer (ELSZ-2Plus, Otsuka Electronics Co., Ltd.) as well as a transmission electron microscope (JEM-2000FX, JEOL).

\section{Preparation of $30 \mathrm{~nm}$ AuNPs}

A 24-mL aliquot of $1.0 \mathrm{wt} \% \mathrm{HAuCl}_{4}$ was added to $180 \mathrm{~mL}$ of $0.26 \mathrm{wt} \%$ sodium citrate and stirred at $353 \mathrm{~K}$ for $20 \mathrm{~min}$. The resulting solution was centrifuged at $8500 \mathrm{rpm}$ at $278 \mathrm{~K}$. The precipitate was redispersed in $30 \mathrm{~mL}$ of ultrapure water, and then the suspension was centrifuged using the same conditions as described above. These procedures were repeated three times. The precipitate that contained no unreacted species was redispersed in ultrapure water. Characterizations using a particle size analyzer and TEM revealed that the produced AuNPs had a mean diameter of $31 \pm 4.0 \mathrm{~nm}$.

\section{Preparation of $12 \mathrm{~nm}$ AuNPs}

An aqueous $0.10 \mathrm{M}$ potassium carbonate solution $(3.0 \mathrm{~mL})$ was added to an aqueous $0.030 \mathrm{wt} \% \mathrm{HAuCl}_{4}$ solution $(100 \mathrm{~mL})$, and the mixture was stirred at $277 \mathrm{~K}$. After adding an aqueous solution of $2.0 \mathrm{wt} \%$ sodium ascorbate $(2.6 \mathrm{~mL})$, the solution was stirred for $20 \mathrm{~min}$ at $353 \mathrm{~K}$. The resulting AuNPs had a mean diameter of $12 \pm 2.0 \mathrm{~nm}$.

\section{Preparation of $2 \mathrm{~nm}$ AuNPs}

An aqueous $0.10 \mathrm{M}$ potassium carbonate solution $(2.0 \mathrm{~mL})$ was added to an aqueous $0.030 \mathrm{wt} \% \mathrm{HAuCl}_{4}$ solution $(100 \mathrm{~mL})$, and the mixture was stirred at $277 \mathrm{~K}$. After slowly adding a $13 \mathrm{mM}$ aqueous solution of sodium borohydride $(2.5 \mathrm{~mL})$, the resulting solution was stirred for $20 \mathrm{~min}$. The resulting AuNPs had a mean diameter of $2.4 \pm 0.3 \mathrm{~nm}$.

\section{Formation of AuNP layer on plastic microbeads}

The AuNPs were deposited on a plastic microbead in a single step. $^{2-4,13}$ Fifty milligrams of acrylic resin microbeads and a binder were added to the colloidal gold dispersion; the mixture was then stirred at room temperature for one day. AuNP coverage of a microbead was controlled by changing the amount of binder added to the mixture solution.

After the AuNP-coated microbeads were separated via filtration, they were washed with an ample amount of water and dried in vacuo. The surfaces of the microbeads were imaged using field-emission scanning electron microscopy (FE-SEM; S-4700, Hitachi) at an applied voltage of $10 \mathrm{kV}$. The AuNP coverage of a microbead was estimated from the average percentages of the bright and dark areas in the enlarged images of ten different beads, since there were clearly two different contrasting bright (AuNP) and dark portions (resin), based on their conductivities.

\section{Dark-field observations}

DFM detects only the light scattered by a sample, while directly transmitted light is blocked using a dark-field condenser, as shown in Scheme 1. Dark-field light-scattering images were acquired using an optical microscope (Eclipse 80i, Nikon) with a dark-field condenser, a 100-W halogen lamp, and a CCD

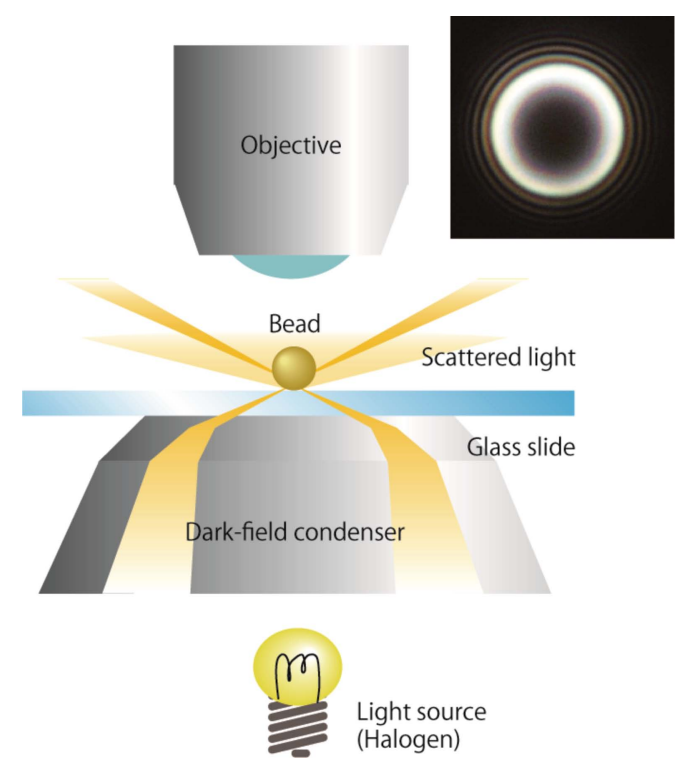

Scheme 1 Schematic illustration of the dark-field microscope setup. The bead insert was placed directly onto the glass slide. The inset shows a dark field image of a bare microbead.

camera. Scattering spectra were obtained using a miniature grating spectrometer (USB4000, Ocean Optics), which was connected to the microscope using an optical fiber. The lightscattering spectra were corrected for spectral variations in the system response, and the white-light intensity distribution (main intensity, $600 \mathrm{~nm}$ ) through division by bright-field spectra was recorded through the sample. The collection volume based on the cross-sectional area (approximately $10 \mu \mathrm{m}^{2}$ ) for the combination of the $100 \times$ objective (NA 0.9) and optical fiber (core diameter, $400 \mu \mathrm{m}$ ) was used in this study. ${ }^{11,12,14}$

\section{Results and Discussion}

SEM observation of a AuNP layer formed on a microbead

A SEM image of a bare microbead shows a smooth acrylic resin surface, as shown in Fig. 1a. We attempted gold-coating of a microbead by using a AuNP dispersion and an appropriate amount of binder molecules. Figure 1 shows SEM images of microbeads that were coated using AuNPs with mean diameters of (b) $2 \mathrm{~nm}$, (c) $12 \mathrm{~nm}$, and (d) $30 \mathrm{~nm}$. Every microbead was almost fully coated with AuNPs, and featured a uniform surface without any overlap of the AuNPs. ${ }^{13}$

We previously reported that the formation of a uniform AuNP monolayer on a microbead depended strongly on the adsorption state of binders to the substrate, and was achieved via the formation of a monolayer of the binder molecules on the microbead..$^{2-4}$ The binder monolayer was obtained on the substrate by controlling the amount of binder in the reaction solution, regardless of the type of binder. The binder in the monolayer on the microbead readily allows for the formation of a single AuNP layer through spontaneous binding. Therefore, it is possible to form a uniform AuNP layer by adding an appropriate amount of AuNP dispersion based on the surface area of the microbead.

Evaluation of metal layer thickness of microbeads using DFM A bare microbead shows a very bright white glow with interference light in the DFM image, as shown in Fig. 2Aa. 
Although we could not find a clear difference among the DFM images of the AuNP-covered microbeads (Figs. 2Ab-2Ac), the light-scattering intensity drastically decreased with an increase in the mean diameter of AuNPs. It is suggested that the intensity is closely correlated to the diameter of the AuNPs; in other words, the intensity strongly reflects the thickness of a gold layer formed on a microbead, since the microbeads have been almost fully covered with AuNPs uniformly without any overlap of the AuNPs (refer to Fig. 1).

The light-scattering spectra were normalized by the spectrum of the incident light source, which had a white-light intensity distribution of the incident light $\left(I_{0}\right.$, shown as a gray spectrum in Fig. 2A), as shown in Fig. 2B. Removal of the incident light components from the obtained spectrum clarified the lightscattering characteristics of the bead. The respective normalized intensity $\left(I / I_{0}\right)$ depended clearly on the thickness of the gold layer, based on the AuNP diameter, over a wide range of wavelengths. When the light $\left(L_{0}\right)$ from the source was incident at an angle of $\theta_{\mathrm{i}}$ to a bare microbead, the incident light $\left(L_{1}\right)$ and reflected light $\left(L_{0}{ }^{\prime}\right)$ originated at the air/solid interface on the microbead, owing to the different refractive indexes of the acrylic resin (1.5) and the air surrounding the bead (1.0), as shown in Scheme 2a. At this time, the AuNP layer on a microbead inhibited the incidence of light. We also found
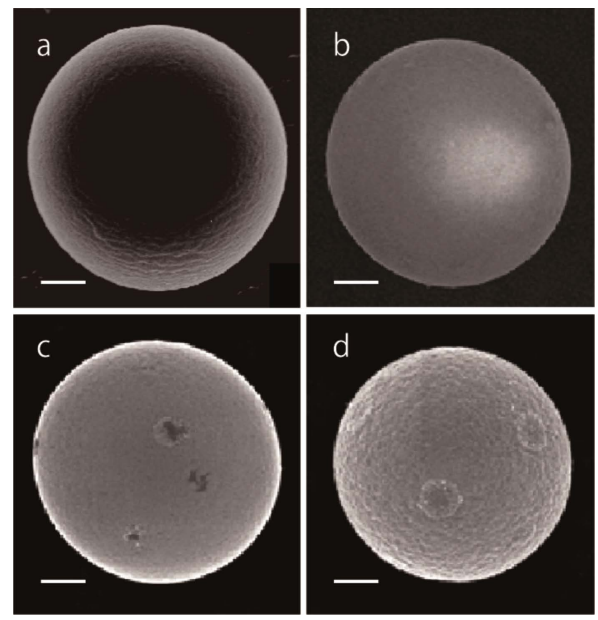

Fig. 1 SEM images of (a) a bare microbead and microbeads fully coated using respective AuNPs with mean diameters of (b) $2 \mathrm{~nm}$, (c) $12 \mathrm{~nm}$, and (d) $30 \mathrm{~nm}$. The bar scale represents $1 \mu \mathrm{m}$. periodic spikes based on the whispering gallery mode (WGM) in the normalized spectrum of a single bead after removal of the components of the incident light. ${ }^{15}$ An expression of the
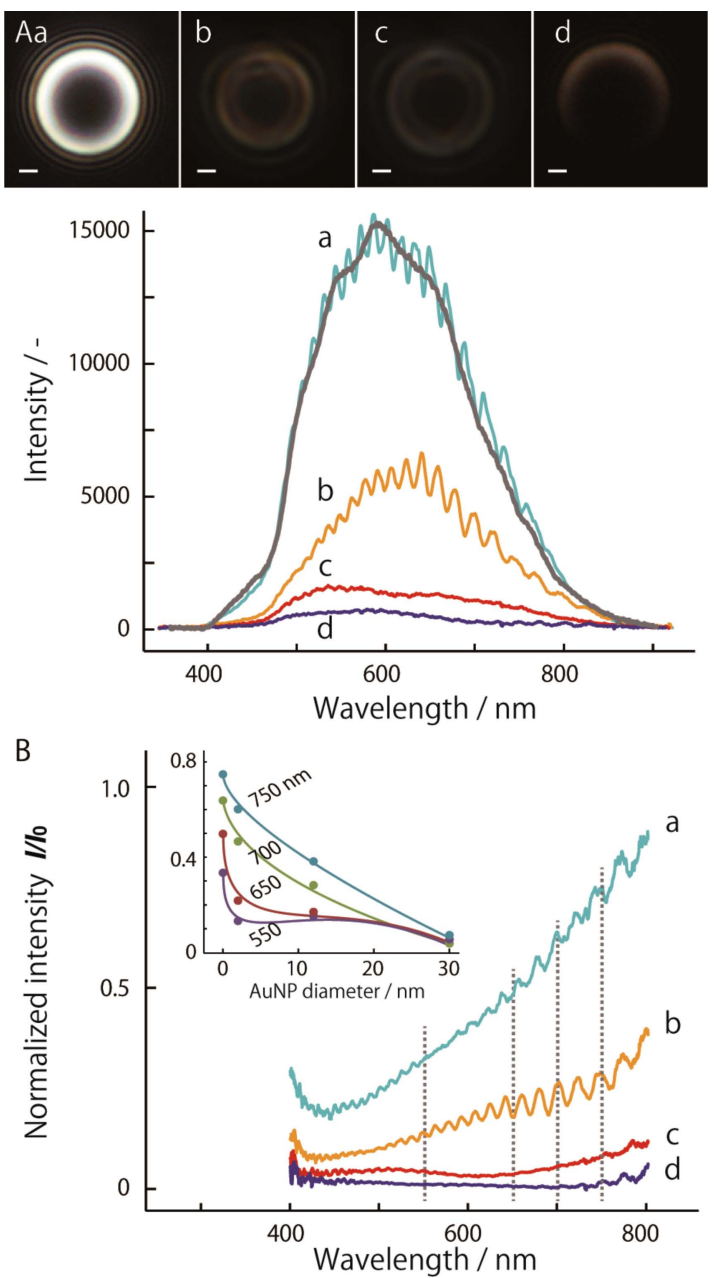

Fig. 2 (A) DFM images and light-scattering spectra of (a) a bare microbead and microbeads fully covered with respective AuNPs with mean diameters of (b) $2 \mathrm{~nm}$, (c) $12 \mathrm{~nm}$, and (d) $30 \mathrm{~nm}$. The gray spectrum displays the white-light intensity distribution of the incident. (B) The normalized intensity $\left(I / I_{0}\right)$ obtained from the light-scattering spectrum divided by the incident spectrum. The inset shows the dependencies of the $I / I_{0}$ value of the microbead on the diameters of AuNPs at the respective wavelengths between 550 and $750 \mathrm{~nm}$. a Air (refractive index 1.0)

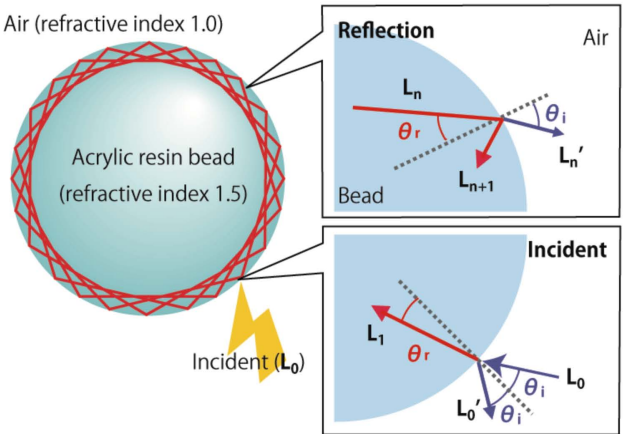

b

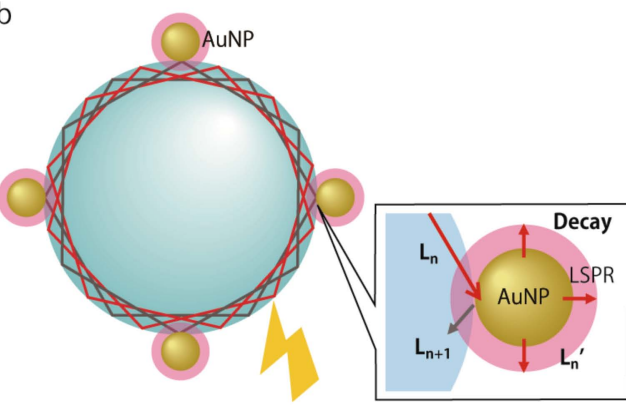

Scheme 2 Model illustrations of the light-scattering characteristics of (a) a bare microbead and (b) a microbead covered with AuNPs. The insets show models of the light reflection of the internal microbead. 
WGM in beads can be explained through the reflection of incident light $\left(L_{1}\right)$ at the air/solid interface that had originated at the former gas/solid interface. Such repeatedly reflected light $\left(L_{n+1}, n>0\right)$ is eventually confined, and propagates inside the acrylic bead, which yields the observed periodic spikes based on the WGM in the spectrum of a single bead. ${ }^{16-19}$ On the other hand, the resonance mode was inhibited by the existence of AuNPs on the surface of a microbead. We observed a decrease in the periodic spikes as the diameter of AuNPs on a microbead increased. This implies that the light incident onto the gas/solid interface is absorbed by the AuNPs, as shown in Scheme $2 b{ }^{20}$ To evaluate the thickness of the gold layer on microbeads, we focused on the dependence of the normalized intensity $\left(I / I_{0}\right)$ on the diameter of AuNPs, as shown in Fig. 2B. The $I / I_{0}$ value decreased as the thickness of the gold layer on a microbead increased in the $550-750 \mathrm{~nm}$ wavelength range. However, we found that the localized surface plasmon resonance (LSPR) of the thin gold layer at wavelengths less than $650 \mathrm{~nm}$ had an effect, as shown in the inset of Fig. 2B. At wavelengths greater than $700 \mathrm{~nm}$, a good correlation between the $I / I_{0}$ value and the AuNP-coated microbead was found.

\section{Estimation of metal coverage of microbeads using DFM}

We also attempted to estimate the surface coverage of a microbead coated using the AuNPs with a mean diameter of $30 \mathrm{~nm}$. SEM images of the AuNP-coated microbeads are shown in Fig. 3. A fully AuNP-covered microbead (a) has a uniform surface (see Fig. 1d). We found that densely adsorbed AuNPs had a mean diameter of $30 \mathrm{~nm}$ on a microbead without any overlap of the AuNPs. Although the micrograph (b) also shows a uniform surface of a microbead, there were clearly two different contrasting bright and dark portions. The AuNP coverage of a microbead was estimated to be $75 \%$ in the enlarged image, since a bright dot attributed to a AuNP was observed on a bare microbead, which was observed as a dark portion. As the AuNP coverage decreased, the bare portions of the microbead surface increased. These results indicate that a single layer of AuNPs formed on the microbead without any overlapping.

A bare microbead shows a very bright white glow with interference light in the dark-field image, as shown in Fig. 4Aa. The brightness of the microbeads decreased as the AuNP coverage on a microbead increased. In the same way, the lightscattering intensity decreased as the AuNP coverage increased, and then became one fifth of that of the bare microbead with over $75 \%$ coverage, as shown in Fig. 4B. On the other hand, we found remarkable periodic spikes in the light-scattering spectrum of the bare microbead (a). Although the periodic spikes were also observed at 50\% AuNP coverage (c), they were hardly observed at over $75 \%$ AuNP coverage.

The incident light $\left(L_{1}=L_{0}-L_{0}{ }^{\prime}, L_{1}>L_{0}{ }^{\prime}\right)$ is reflected repeatedly at the gas/solid interface in the bead, since there is a difference in the refractive indexes between the acrylic resin and air surrounding around the bead, as shown in Scheme 2a. The light incident $\left(L_{\mathrm{n}}\right)$ onto the gas/solid interface generates reflected light $\left(L_{\mathrm{n}+1}=L_{\mathrm{n}}-L_{\mathrm{n}}{ }^{\prime}, L_{\mathrm{n}+1}>L_{\mathrm{n}}{ }^{\prime}\right)$, and is eventually confined and circulates inside the bead. When the circumference of the bead becomes an integral multiple of the wavelength of light, the light circulating in the bead resonates with a strongly aligned phase. The light intensity is strongly enhanced at specific wavelengths, and is therefore observed as periodic spikes in the spectrum of a single bead in the gas phase. The resonance mode, based on the WGM, can be observed in spectra (a) - (c) of Fig. 4B. On the other hand, the resonance mode decreases as the AuNP coverage of a microbead increases, and then
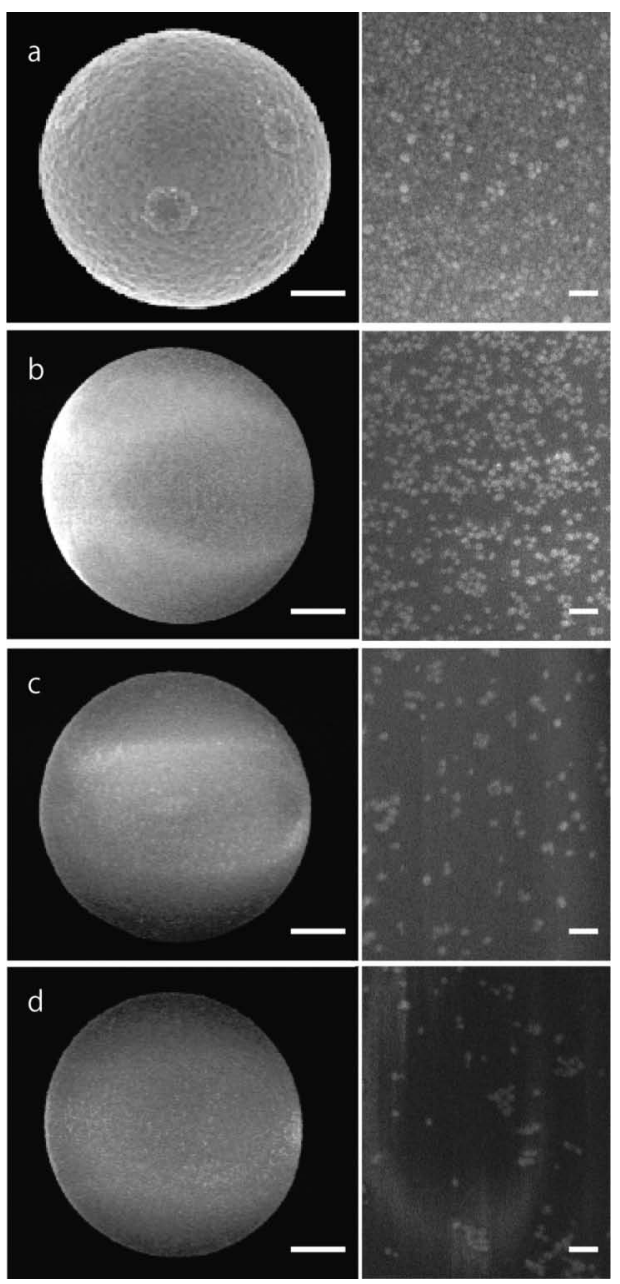

Fig. 3 SEM images and enlarged images of a microbead covered using $30 \mathrm{~nm}$ AuNPs. The scale bars represent $1 \mu \mathrm{m}$ (left) and $100 \mathrm{~nm}$ (right), respectively. Estimated surface coverage: (a) 100\%, (b) $75 \%$, (c) $50 \%$, and (d) $25 \%$.

disappears completely at $100 \%$ coverage. The incident light generates the reflected light $\left(L_{\mathrm{n}+1}=L_{\mathrm{n}}-L_{\mathrm{n}}{ }^{\prime}\right)$ based on the refractive index at the gas/solid interface. In this case, $L_{\mathrm{n}}{ }^{\prime}$ is strongly affected by the presence of AuNP. When AuNPs are present on the surface of a microbead, the incident light $\left(L_{\mathrm{n}}\right)$ at the gas/solid interface penetrates to the outside of the bead through the AuNPs, which generates the LSPR $\left(L_{\mathrm{n}}{ }^{\prime}\right)$; therefore, the reflected light $\left(L_{\mathrm{n}+1}\right)$ decreases significantly and disappears, as shown in Scheme 2b. There are two plausible explanations for the light-scattering properties of the AuNP-coated microbead. One explanation is based on a reduction of the incident light intensity $\left(L_{1}\right)$ from the outside of the bead owing to reflection by the covered AuNP $\left(L_{1}<<L_{0}{ }^{\prime}\right)$. The other explanation is based on the decrease in the reflectivity $\left(L_{n+1} / L_{n}\right)$ at the gas/solid interface in the internal bead due to the expression of the LSPR, which leads to an increase in $L_{n}{ }^{\prime}$. Therefore, the light-scattering intensity of the bead depends strongly on the $L_{n+1}$, which associates closely with the WGM.

As mentioned above, we must deal with the $I / I_{0}$ value in a longer wavelength region without the effect of LSPR, and take into account the effect of the WGM in order to evaluate the AuNP coverage of a microbead. We have obtained a good correlation between the $I / I_{0}$ value and the AuNP-coated microbeads at a wavelength of $700 \mathrm{~nm}$, as shown in Fig. 4C. 


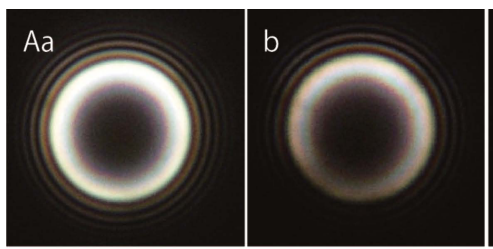

B

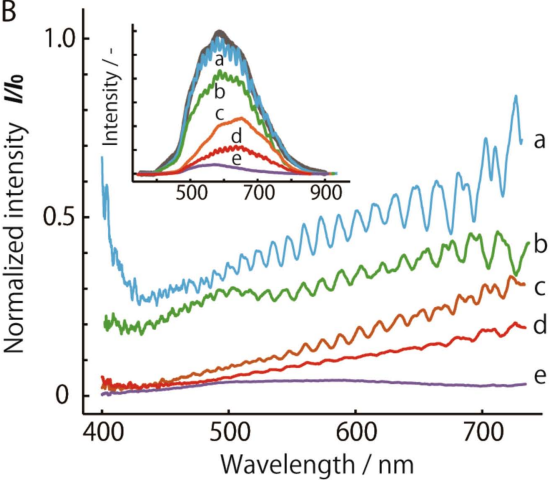

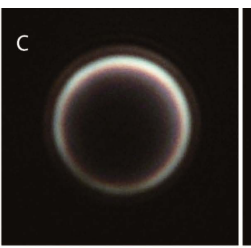

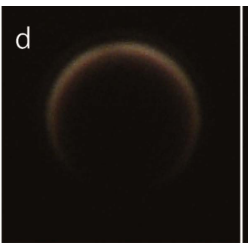

C

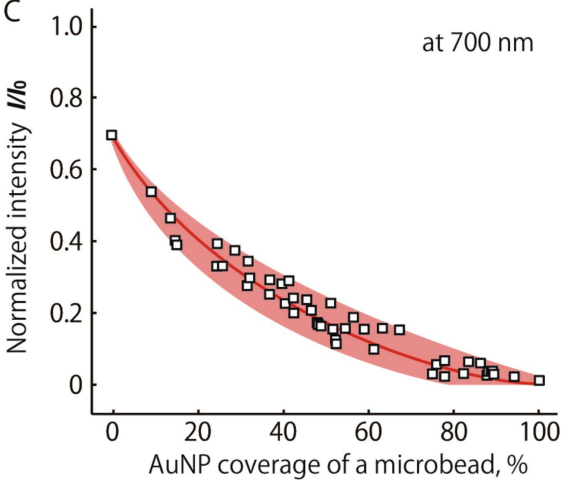

Fig. 4 (A) DFM images and (B) the normalized intensity $\left(I / I_{0}\right)$ obtained from the light-scattering spectrum divided by the incident spectrum. Estimated surface coverage: (a) $0 \%$, (b) $25 \%$, (c) $50 \%$, (d) $75 \%$, and (e) $100 \%$. (C) The dependence of the $I / I_{0}$ value at $700 \mathrm{~nm}$ of the microbead on the AuNP coverage.

Recently, we also reported an estimation of the silver-NP coverage of microbeads, and found a good correlation between the $I / I_{0}$ value at $700 \mathrm{~nm}$ and the silver-NP coverage. ${ }^{15}$ The present method is useful to evaluate not only the metal coverage of microbeads regardless of the metal species, but also the metal film thickness. Therefore, we believe that our approach represents a breakthrough in evaluating the coverage and thickness of a metal-NP layer formed on a microbead.

\section{Conclusions}

We successfully developed a simple evaluation method to estimate the coverage of AuNP coatings on microbeads based on light-scattering. For a more accurate evaluation of the coverage, not only do the effects of the WGM and LSPR need to be examined, but also the size and metal species of the NPs. Although such an examination is necessary, we believe that our approach represents a breakthrough.

\section{Acknowledgements}

We gratefully acknowledge financial support provided by the Ministry of Agriculture, Forestry, and Fisheries through a Science and Technology Research Promotion Program for agriculture, forestry, fisheries, and the food industry. We also acknowledge financial support from the Japan Society for the Promotion of Science (JSPS) through a Grant-in-Aid for Scientific Research (B) (KAKENHI 25288069) and a Grant-inAid for Challenging Exploratory Research (KAKENHI 26620072, 26620123).

\section{References}

1. P. Tierno and W. A. Goedel, J. Phys. Chem. B, 2006, 110, 3043.

2. S. Tokonami, S. Shirai, I. Ota, N. Shibutani, Y. Yamamoto, H.
Shiigi, and T. Nagaoka, J. Electrochem. Soc., 2011, 158, D689.

3. Y. Yamamoto, S. Takeda, H. Shiigi, and T. Nagaoka, J. Electrochem. Soc., 2007, 154, D462.

4. S. Tokonami, Y. Yamamoto, Y. Mizutani, I. Ota, H. Shiigi, and T. Nagaoka, J. Electrochem. Soc., 2009, 156, D558.

5. C. A. Foss, G. L. Hornyak, J. A. Stockert, and C. R. Martin, J. Phys. Chem., 1994, 98, 2963.

6. P. Alivisatos, Nat. Biotechnol., 2004, 22, 47.

7. H. Nakao, H. Shiigi, Y. Yamamoto, S. Tokonami, T. Nagaoka, S. Sugiyama, and T. Ohtani, Nano Lett., 2003, 3, 1391.

8. H. Shiigi, S. Tokonami, H. Yakabe, and T. Nagaoka, J. Am. Chem. Soc., 2005, 127, 3280.

9. H. Shiigi and T. Nagaoka, Anal. Sci., 2014, 30, 89.

10. H. Shiigi, T. Kinoshita, N. Shibutani, T. Nishino, and T. Nagaoka, Anal. Chem., 2014, 86, 4977.

11. H. Shiigi, M. Fukuda, T. Tono, K. Takada, T. Okada, L. Q. Dung, Y. Hatsuoka, T. Kinoshita, M. Takai, S. Tokonami, H. Nakao, T. Nishino, Y. Yamamoto, and T. Nagaoka, Chem. Commun., 2014, 50, 6252.

12. H. Shiigi, T. Kinoshita, M. Fukuda, L. Q. Dung, T. Nishino, and T. Nagaoka, Anal. Chem., 2015, 87, 4042.

13. H. Shiigi, S. Shirai, T. Fujita, H. Morishita, Y. Yamamoto, T. Nishino, S. Tokonami, H. Nakao, and T. Nagaoka, J. Electrochem. Soc., 2013, 160, H630.

14. T. Kobori, J. Watanabe, and H. Nakao, Anal. Sci., 2012, 28, 61.

15. H. Shiigi, S. Kimura, T. Fujita, and T. Nagaoka, Anal. Sci., 2015, 31, 577.

16. T. Mitsui, Y. Wakayama, T. Onodera, Y. Takaya, and H. Oikawa, Nano Lett., 2008, 8, 853.

17. S. Ishizaka, Y. Suzuki, and N. Kitamura, Phys. Chem. Chem. Phys., 2010, 12, 9852.

18. Q. J. Wang, C. Yan, N. Yu, J. Unterhinninghofen, J. Wiersig, C. Pflügl, L. Diehl, T. Edamura, M. Yamanishi, H. Kan, and F. Capasso, Proc. Natl. Acad. Sci. U. S. A., 2010, 107, 22407.

19. L. Shi, R. Fenollosa, T. U. Tuzer, and F. Meseguer, ACS Photonics, 2014, 1, 408.

20. A. Panáček, L. Kvítek, R. Prucek, M. Kolář, R. Večeřová, N. Pizúrová, V. K. Sharma, T. Nevěčná, and R. Zbořil, J. Phys. Chem. B, 2006, 110, 16248. 